\title{
Hematological Manifestations among Patients with Rheumatic Diseases
}

\author{
Alina Klein $^{\mathrm{a}}$ Yair Molad ${ }^{\mathrm{b}, \mathrm{c}}$

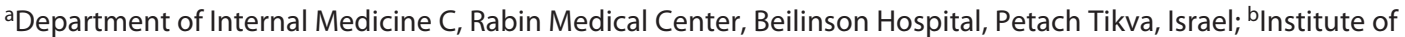 \\ Rheumatology, Rabin Medical Center, Beilinson Hospital, Petach Tikva, Israel; 'Sackler Faculty of Medicine, Tel Aviv \\ University, Tel Aviv, Israel
}

\section{Keywords}

Thrombocytopenia · Anemia · Leukopenia - Thrombosis · Thrombotic microangiopathy · Rheumatic diseases . Arthritis - Vasculitis - Systemic lupus erythematosus · Antiphospholipid syndrome

\section{Abstract \\ Background: Rheumatic diseases have many hematological manifestations. Blood dyscrasias and other hematological abnormalities are sometimes the first sign of rheumatic dis- ease. In addition, novel antirheumatic biological agents may cause cytopenias. Summary: The aim of this review was to discuss cytopenias caused by systemic lupus erythematosus and antirheumatic drugs, Felty's syndrome in rheumatoid ar- thritis, and autoimmune hemolytic anemia, thrombosis, and thrombotic microangiopathies related to rheumatological conditions such as catastrophic antiphospholipid syndrome and scleroderma renal crisis. Key Message: The differential diagnosis of various hematological disorders should include rheumatic autoimmune diseases among other causes of blood cell and hemostasis abnormalities. It is crucial that he- matologists be aware of these presentations so that they are diagnosed and treated in a timely manner.}

\section{Introduction}

Rheumatic autoimmune diseases (RADs) such as rheumatoid arthritis (RA) and systemic lupus erythematosus (SLE) are characterized by multi-organ involvement along with the production of pathogenic autoantibodies. RADs are often associated with hematological manifestations which sometimes serve as the presenting sign, such as cytopenia in SLE [1], neutropenia in Felty's syndrome, and thrombocytopenia in antiphospholipid syndrome (APS). The differential diagnosis of various hematological disorders should include RAD among other causes of blood cell and hemostasis abnormalities. In addition, novel antirheumatic biological agents may cause cytopenias. The purpose of this review was to summarize the current data on the common hematological manifestations in patients with RADs.

\section{Leukopenia in Systemic Lupus Erythematosus}

Hemolytic anemia, leukopenia, lymphopenia, and immune-mediated thrombocytopenia are frequently seen in patients with SLE, either at disease presentation or during flares $[1,2]$, and they are considered a criterion for its

karger@karger.com
www.karger.com/aha
Karger $V^{\prime /}$


classification [3, 4]. Among the clinical criteria for SLE, the American College of Rheumatology (ACR) [3] includes leukopenia, defined as a white blood cell count of less than $4,000 / \mathrm{mm}^{3}$ or lymphopenia of less than $1,500 /$ $\mathrm{mm}^{3}$ on at least 2 occasions. The Systemic Lupus Collaborating Clinics (SLICC) [4] includes leukopenia, defined as a leukocyte count of less than $4,000 / \mathrm{mm}^{3}$ or lymphopenia of less than $1,000 / \mathrm{mm}^{3}$ at least once $[1,2]$.

Leukopenia presents in approximately $50 \%$ of the patients with SLE, and some observational studies reported leukocyte abnormalities in up to $75 \%$ of the patients at some point during the disease course [5]. Both granulocyte and lymphocyte lineage can be affected, but lymphopenia is more common and correlates with disease activity [5]. Dias et al. [6], in a study of 124 patients with SLE, reported severe lymphopenia in $4 \%$ of the patients and severe neutropenia $\left(<1,000 / \mathrm{mm}^{3}\right)$ in $0.8 \%$ of the patients. Rivero et al. [7] found that of the 158 patients with active untreated SLE, $10 \%$ had severe lymphopenia. Although it is debatable whether lymphopenia is a cause or a consequence of autoimmunity that involves the bone marrow, in the presence of thrombocytopenia and other lineage damage, it is reasonable to assume that it is a result of autoimmune processes [8]. The pathogenesis of lymphopenia involves autoimmunity by antilymphocyte antibodies, circulating lymphotoxic antibodies such as cold-reactive IgM, anti-Ro/SS-A antibodies, and accelerated lymphocyte apoptosis [9]. The mechanisms responsible for neutropenia are unclear, but it is thought to be related to marrow suppression by $\mathrm{T}$ lymphocytes, peripheral destruction by antineutrophil antibodies, and complementactivating anti-neutrophil IgG autoantibodies. Anti-Ro/ SS-A antibodies have also been found to be associated with the presence of neutropenia in SLE [9].

Besides disease activity, the differential diagnosis of leukopenia in patients with SLE includes drug-induced leukopenia as well as non-SLE causes such as infections or malignancy. Thus, a full workup is warranted, including a comprehensive medication history, as detailed below. The evaluation should comprise at least complete blood count and blood smear to determine the leukocyte morphology. Bone marrow smear should be considered, especially in the presence of pancytopenia or in any case in which bone marrow failure is suspected, as bone marrow is a target organ for SLE activity, and SLE increases the risk of development of lymphoma. In patients with isolated persistent leukopenia without a history of use of offending drugs, viral etiology such as cytomegalovirus, parvovirus B19, and human immunodeficiency virus, should be ruled out $[5,10]$. Neutropenia was reported to occur in $20-47 \%$ of the patients with SLE $[2,6,11,12]$. A recent large multicentric registry (Lupus BioBank of the upper Rhein) found a prevalence of $21 \%$ of neutropenia in Caucasian SLE that was significantly associated with thrombocytopenia (OR 3.68 [2.58-5.25], $p<0.0001$ ), lymphopenia (OR 3.34 [2.37-4.72], $p<0.0001$ ), low complement C3 (OR 1.83 [1.29-2.59], $p=0.0006$ ), and low C4 (OR 1.62 [1.15-2.29], $p=0.006$ ) and positive Coombs' test (OR 2.91 [1.96-4.32], $p<0.0001$ ) [13]. Among other causes of neutropenia in SLE patients, benign ethnic (familial) neutropenia, which is one of the most common causes of chronic neutropenia seen in individuals of African, Middle Eastern, and west Indian descent [14], should be excluded before attributing lupus as the cause of neutropenia.

The recent review of Carli et al. [15] on the clinical impact of leukopenia on the risk of infections showed that the data on lymphopenia of less than $1,000 / \mathrm{mm}^{3}$ were inconsistent among different studies. Some reported an increased risk of infection with a low absolute lymphocyte count, especially infection of the urinary tract, skin and soft tissue, pneumonia, and bacteremia, whereas others did not. Moderate and severe neutropenia of less than $1,000 / \mathrm{mm}^{3}$ and $500 / \mathrm{mm}^{3}$, respectively, was associated with severe infections in some studies and with no increased risk in others [15].

Information on treatment with granulocyte colonystimulating factor (G-CSF) derives mainly from case reports and case series. G-CSF seems to be beneficial especially in the context of neutropenia complicated by infection, as it quickly raises the neutrophil count. However, G-CSF agents have been associated with SLE flares in these patients, so the dose should be minimal, with a target neutrophil count of just above $1,000 / \mathrm{mm}^{3}$ [15-17].

\section{Felty's Syndrome}

Felty's syndrome should be considered a cause of neutropenia in patients with RA. Classically, Felty's syndrome is manifested by the triad of RA, neutropenia, and splenomegaly and complicates up to $3 \%$ of the cases of RA. However, the only diagnostic criterion of Felty's syndrome is persistent idiopathic neutropenia with an absolute neutrophil count of less than $1,500 / \mathrm{mm}^{3}$, such that splenomegaly and severe joint involvement may be absent [18]. Usually, Felty's syndrome develops years after the diagnosis of RA, although in rare instances it may develop before. In these cases, the diagnosis of RA is established by a finding of elevated rheumatoid factor and/or 
anti-cyclic citrullinated peptide antibody in patients with polyarthritis and unexplained neutropenia [19].

The clinical features of Felty's syndrome differ among patients, with a large variation in the severity of the articular disease, the duration of RA before the appearance of neutropenia, and the neutrophil count. In general, patients with Felty's syndrome have more frequent extra-articular manifestations of rheumatoid nodules, lymphadenopathy, hepatomegaly, anemia, and thrombocytopenia than patients with RA without Felty's syndrome. The syndrome also has a strong association with HLA-DR4 [20]. The principle mechanisms responsible for neutropenia in Felty's syndrome are similar to those in SLE, namely, immunologic inhibition of bone marrow granulopoiesis, inhibition of G-CSF-induced maturation, and increased peripheral destruction. Increased sequestration in the spleen has been reported as well [21, 22]. The most common bone marrow findings are myeloid hyperplasia and maturation arrest. However, the bone marrow may also appear nor$\mathrm{mal}$, and in rare cases, there may be bone marrow hypocellularity and reduced myelopoiesis. Although bone marrow findings are not specific, aspiration is recommended as part of the evaluation, mainly to rule out malignancy [18]. Neutropenia in Felty's syndrome is clinically relevant as it exposes patients to serious infections, usually when the absolute neutrophil count is less than $1,000 / \mathrm{mm}^{3}$. The skin, mouth, and pulmonary tract are the most frequent organs affected by infections; Staphylococcus aureus, Streptococcus, Gram-negative enteric bacteria, and fungi are the most frequent pathogens $[18,20]$.

The treatment of Felty's syndrome has never been studied in randomized controlled trials, and the available data are derived from case reports. Felty's syndrome with recurrent infections is usually treated with methotrexate, and G-CSF is added when the absolute neutrophil count is less than $1,000 / \mathrm{mm}^{3}[18]$. In some patients, but not all, refractory Felty's syndrome may respond to rituximab. The response varies, suggesting that different mechanisms may be responsible for the neutropenia [22, 23].

\section{Thrombocytopenia in Systemic Lupus Erythematosus and Antiphospholipid Syndrome}

Thrombocytopenia is a well-known manifestation of such autoimmune diseases as SLE, APS, and Felty's syndrome. Thrombocytopenia may also be secondary to antirheumatic drug use $[21,24]$. Among patients with SLE, up to $25 \%$ have thrombocytopenia of less than $100,000 /$ $\mathrm{mm}^{3}$, and approximately $10 \%$ have severe thrombocyto-

Hematological Manifestations in Rheumatic Diseases penia. In about $15 \%$ of the patients, isolated thrombocytopenia precedes the diagnosis of SLE by several years [5].

Thrombocytopenia in SLE can present in several ways. In some patients, it is chronic and usually asymptomatic, whereas in others, it may be acute and severe, occurring as part of disease exacerbation and requiring immediate treatment [1]. The acute form responds better to glucocorticoids than the chronic and less severe form [9]. Thrombocytopenia is a bad prognostic marker in SLE and is related to greater morbidity and mortality [5]. A multiethnic cohort study of 616 patients with SLE found that thrombocytopenia was associated with disease activity and renal, neurological, and hematological involvement. On multivariate analysis, thrombocytopenia of less than $100,000 / \mathrm{mm}^{3}$ was a predictor of mortality [25]. Except in cases of drug-induced lupus and pseudothrombocytopenia, the thrombocytopenia in SLE is mainly immune-mediated, involving antiplatelet antibodies. It is also associated with antiphospholipid antibodies, which should be tested in all patients with SLE. In patients with SLE or APS treated with heparin or low-molecular-weight heparin, heparin-induced thrombocytopenia should be ruled out because its clinical presentation can mimic thrombotic angiopathy, including catastrophic APS (CAPS) $[5,9]$.

The decision to treat thrombocytopenia is based on several considerations including platelet count, bleeding risk, and active bleeding. According to current recommendations, a platelet count below $30,000-50,000 / \mathrm{mm}^{3}$ will usually require treatment, as bleeding risk increases [26]. Glucocorticoids are the first-line therapy, administered either orally or in more severe cases, as an intravenous pulse. Intravenous immunoglobulins (IVIGs) can be added in cases of glucocorticoids - refractory thrombocytopenia - and/or life-threatening bleeding. Most patients will respond initially to glucocorticoids but will not achieve long-term remission. Danazol and hydroxychloroquine (Plaquenil) can be added as glucocorticoids sparing agents - as was shown by Arnal et al. [27]. In this case series of 59 patients with SLE and immune thrombocytopenia, a combination of danazol or Plaquenil with glucocorticoids achieved higher rates of sustained responses compared to glucocorticoids alone (50 and 64\% respectively), and allowed glucocorticoids to be tapered down and withdrawn [27].

Other immunosuppressive options for the treatment of thrombocytopenia in SLE include azathioprine, mycophenolate, cyclophosphamide, cyclosporine, and vincristine $[9,28]$. The newer, biological agents that have been studied are rituximab, belimumab, and thrombopoietin (TPO) mimetics. The results of a 2014 review of 6 cohort 
studies of rituximab treatment of SLE-related thrombocytopenia yielded a range of complete or partial responses, from 50 to $92 \%$ of the patients [29]. More recently, a cohort study including 44 patients with SLE-related thrombocytopenia reported an overall response rate of $100 \%$, with complete response in $56.8 \%$ of the patients and partial response in $34 \%$. Relapse occurred in $40 \%$ of each group [30]. Thus, it seems that rituximab is effective in achieving good long-term results in SLE-related thrombocytopenia, and it is frequently used, especially in patients who do not respond to glucocorticoids [26].

The study of TPO mimetics in SLE-related thrombocytopenia was prompted by reports of its beneficial effect in primary immune thrombocytopenic purpura [26]. A recent single-center study found that all 12 patients with SLE-related thrombocytopenia achieved a sustained response to treatment with eltrombopag on top of glucocorticoids and other immunosuppressants, with no evidence of serious adverse events such as thromboembolism [31]. Another case series study of 4 patients with SLE and glucocorticoids - refractory thrombocytopenia - including 1 with concurrent APS, found that in 3 cases, TPO mimetics improved platelet counts and prevented bleeding events. No SLE flares or new thrombotic events were noted [32]. Nevertheless, TPO mimetics are usually not recommended for patients with antiphospholipid antibodies because of an increased risk of thrombotic events. Thus, based on the existing data, TPO mimetics may be considered for patients with SLE and refractory thrombocytopenia.

Thrombocytopenia is one of the non-criteria manifestations of primary APS (with no evidence of SLE). It occurs in $22-42 \%$ of the patients and is usually moderate (platelet count above $50,000 / \mathrm{mm}^{3}$ ). Rarely does thrombocytopenia in APS have clinical significance or require treatment. The exception is a platelet count below 30,000/ $\mathrm{mm}^{3}$ with symptomatic bleeding, which warrants treatment as in immune thrombocytopenic purpura [33].

\section{Autoimmune Hemolytic Anemia in Systemic Lupus Erythematosus}

Autoimmune hemolytic anemia (AIHA) [34] is one of the clinical criteria of SLE according to the classifications of both the ACR and SLICC [3,4]. It occurs as part of SLE flare [28] with or without leukopenia and thrombocytopenia, as in Evan's syndrome [35]. According to a retrospective single-center study investigating the clinical data of 101 patients with AIHA, SLE was the secondary cause in 64\% [34]. The prevalence of AIHA in SLE varies be- tween 3 and $8 \%$ among studies. AIHA may precede SLE diagnosis by several years or coincide with SLE onset [36, 37]. In patients with SLE, the anti-erythrocyte antibodies are usually warm-type IgG, meaning that their reactivity to erythrocytes is better at warm temperatures, with optimum reactivity at body temperature. The erythrocytes coated by warm IgG antibodies undergo membrane changes as they pass through the spleen, and the resulting spherocytes are removed by phagocytosis [36]. The direct antiglobulin test is used to screen for erythrocyte antibodies, and positive results are common in patients with SLE. In 1 cohort study of 373 patients with SLE, $12.8 \%$ had a positive direct antiglobulin test; however, only half of them had overt hemolysis [38].

Glucocorticoid treatment of AIHA has been associated with a $90 \%$ response rate within 3 weeks. However, most patients require maintenance therapy [39]. Secondline therapeutic agents include azathioprine, IVIG, danazol, and rituximab. As there is no consensus regarding the preferred second-line agent, treatment is tailored to the individual patient $[9,28]$. Rituximab is being increasingly used as a second-line agent because it has the highest response rates [39]. Its efficacy was investigated in a meta-analysis of 21 studies with a total of 409 patients. In the subgroup of 66 patients with secondary warm AIHA, the overall response rate was $72 \%$. The complete response rate was initially $46 \%$, and it increased over 3 months of follow-up. Relapse occurred in more than $30 \%$ of the responders after 3 years, but retreatment with rituximab led to a second remission in most cases [40].

\section{Antirheumatic Drugs and Cytopenias}

As discussed above, blood dyscrasias are not rare in patients with rheumatic diseases, as bone marrow and blood cells can be targets for autoimmune processes. Increasing the complexity of the picture is the potentially adverse effect on blood counts of many of the disease-modifying antirheumatic drugs used for the treatment of inflammatory arthritis such as RA and psoriatic arthritis. In this section, we will focus on the novel biological agents.

Neutropenia caused by tumor necrosis factor alpha inhibitor (TNFi) agents is well described in the literature [41-43]. The mean interval from the onset of therapy with a TNFi to the development of neutropenia is 3 months, but with a wide range of 1 week to 26 months [41]. In cohort studies, an episode of neutropenia of no apparent cause other than TNFi intake occurs in about $14 \%$ of the patients receiving these agents $[41,42]$. Usu- 
ally, TNFi-induced neutropenia is mild to moderate, but in rare cases the absolute neutrophil count may drop to less than $500 / \mathrm{mm}^{3}$, causing life-threatening infections [42]. The absolute neutrophil count usually recovers after cessation of the offending TNFi, and once it rises, the agent can be restarted or switched to another TNFi [42]. Although there is no official recommendation to perform routine blood counts during TNFi treatment, they should be considered [43].

Paradoxically, TNFi agents can induce SLE and/or APS, including immune-mediated cytopenia $[44,45]$. An association of TNFi with antiphospholipid antibody-related thrombosis has been described as well [45]. Lateonset neutropenia (LON), occurring from 1 month to 1 year following rituximab treatment, is a well-known side effect in patients with hematological diseases. It has also been described in patients with $\mathrm{RAD}$, although to a lesser extent. A prospective French registry study of 2,624 patients [46] reported LON in $1.5 \%$ of the total cohort and $1.3 \%$ of the patients with RA. Among the 39 patients with LON, 17 (35\%) had an absolute neutrophil count of less than $1,000 / \mathrm{mm}^{3}$, including 4 with a count of $500 / \mathrm{mm}^{3}$ which resulted in serious infection. Another retrospective study of 168 rituximab-treated patients with RAD found that LON developed in 6.5\%, within a time range of $40-$ 198 days. Most of the affected patients had RA. Four patients (2.3\%) acquired serious infections warranting antibiotic treatment, but all recovered without sequelae. In all patients with LON, the neutrophil count normalized after rituximab was stopped. Treatment with G-CSF shortened the time to neutrophil recovery but did not change the outcomes. Readministration of rituximab after the blood count normalized appeared to be safe [47]. These findings were supported by a retrospective analysis of 209 rheumatic patients in whom the rate of LON was $5 \%$, occurring within a similar time frame of 40-362 days. Six patients, representing $2.8 \%$ of the cohort, had sepsis. In all patients, the neutrophil count recovered after cessation of rituximab. In the 3 patients who did not receive G-CSF, normalization of the neutrophil count took about 20 days. The rate of LON was higher in patients with vasculitis and SLE than in patients with RA [48].

Tofacitinib, a Janus kinase inhibitor used for the treatment of RA, may cause slight changes in blood counts, as shown in phase III and long-term extension studies [49]. In 6 phase III randomized controlled trials that included a total of 4,858 patients, the neutrophil count decreased slightly during the first 24 months, with no cases of severe neutropenia. The lymphocyte count increased in the first 3 months and then decreased gradually and stabilized at 48 months.
Severe lymphopenia, defined as an absolute lymphocyte count below $500 / \mathrm{mm}^{3}$, developed in less than $1 \%$ of the patients, most of whom had lymphopenia before onset of tofacitinib treatment. After drug cessation, the absolute lymphocyte count increased. The decrease in both neutrophils and lymphocytes was dose-dependent and was more pronounced in patients receiving a $10-\mathrm{mg}$ than a 5-mg dose. Hemoglobin levels increased in the first 24 months and were correlated to the decrease in inflammatory markers Creactive protein and erythrocyte sedimentation rate. A dose-adjusted decrease in hemoglobin also occurred, but anemia with clinically significant hemoglobin changes was documented in less than $2 \%$ of the patients [49].

Interleukin-6 (IL-6) receptor inhibitors, such as tocilizumab, are indicated for the treatment of RA and giant cell arteritis (GCA) and can cause both neutropenia and thrombocytopenia [50]. Tocilizumab-induced neutropenia has been attributed to a shift in the neutrophil pool toward margination rather than myelosuppression and was not found to be associated with an increase in serious infections. IL-6 stimulates thrombopoiesis by induction of TPO, such that IL- 6 receptor inhibitors can cause thrombocytopenia. However, the thrombocytopenia in this setting is not associated with serious bleeding events owing to the presence of other, non-IL-6-dependent, pathways [50].

\section{Thrombosis and RAD}

The chronic inflammation that underlies active rheumatic diseases constitutes by itself a hypercoagulable state. SLE, RA, Sjogren's syndrome, Behcet's syndrome, and vasculitis are only a few examples of inflammatory conditions associated with an increased risk of thrombosis [51].

Recent data suggest that arterial and venous thrombotic events may be a feature of small-vessel antineutrophil cytoplasmic antibody-associated vasculitis (AAV), including granulomatosis with polyangiitis (GPA), microscopic polyangiitis (MPA), and eosinophilic granulomatosis with polyangiitis (EGPA) [51, 52]. Specifically, GPA and MPA, and to a lesser extent EGPA, have been associated with an increased risk of ischemic heart disease, more cardiovascular events, and subclinical atherosclerosis affecting peripheral blood vessels $[51,52]$. A retrospective cohort study of 113 patients with AAV and matched controls found that the AAV group had more cardiovascular events over a 3.4-year follow-up, with a hazard ratio of 2.23 [53]. Retrospective cohort studies of venous thromboembolism (VTE) in AAV found VTE 
rates of $6.5-8.2 \%$. Patients with EGPA had the highest rates of venous events relative to patients with MPA and GPA, but the lowest rates of arterial events. The risk was highest with active disease and around flares [52, 54, 55].

Large-vessel vasculitis such as GCA and Takayasu's arteritis involve arteries exclusively and are primarily associated with an increased risk of arterial thrombosis [51, 52]. GCA can present with cranial ischemic strokes and strokes in the territory of the carotid artery and the vertebrobasilar artery [56]. Stroke and transient ischemic attacks occur in patients with Takayasu's arteritis with an estimated prevalence of $16 \%$ [57]. Recent cohort studies showed that large-vessel vasculitis also harbors an increased risk of venous thrombosis, with the highest incidence occurring at the time of GCA diagnosis, supporting a causative association between inflammation and hypercoagulability $[58,59]$.

When the diagnosis of GCA is established, combination therapy with antiplatelets and glucocorticoids is recommended, as it has been found in some studies to reduce cerebrovascular events and visual loss. In Takayasu's arteritis, antiplatelet therapy is safe and has a protective effect against cardiovascular events [51, 52]. The management of thrombotic events in AAV is still controversial because glucocorticoids treatment may increase the risk of cardiovascular events, and data on antiplatelet/anticoagulation therapy and VTE prophylaxis remain sparse.

Psoriatic arthritis and RA are chronic inflammatory diseases that can potentially induce thrombosis. Recent cohort studies of VTE in patients with RA from Taiwan [60] and the USA [61] showed that the patients had a higher prevalence of deep vein thrombosis (DVT) and pulmonary embolism (PE) than the general population. These findings were supported by a prospective study from Sweden wherein 37,000 patients followed up for 13 years were found to have double the rates of DVT and PE than a matched cohort from the general population [62]. Less attention has been directed to the risk of VTE in psoriatic arthritis. A recent study compared the rates of DVT and $\mathrm{PE}$ in 12,084 patients with psoriatic arthritis and 51,756 patients with RA with the general population. Both patient groups had an elevated risk of VTE, but findings were significant only for the patients with RA [63].

Patients with SLE are at increased risk for thrombosis, both arterial and venous, regardless of their antiphospholipid antibody status [64]. Arterial thrombosis in SLE is related to early and accelerated atherosclerosis, leading to more cardiovascular disease that ultimately results in myocardial infraction, stroke, and peripheral vascular disease. Studies have pointed to traditional risk factors of age, hypertension, and obesity, as well as disease-specific risk factors such as systemic inflammation and autoantibody-mediated endothelial dysfunction, cumulative (but not daily) glucocorticoids dose, nephrotic syndrome, and presence of anti-Ro/SS-A and anti-La/SS-B antibodies, which are associated with cardiovascular damage and related mortality [64, 65]. A prospective 5-year follow-up study of 219 patients with recent-onset SLE found that $16 \%$ had a thrombotic event during the study period: $3.5 \%$ arterial thrombosis and $12.5 \%$ venous thrombosis. Most of the patients with a thrombotic event (69\%) tested negative for lupus anticoagulant (LAC). More than half the events (57\%) occurred in the first year after diagnosis. Venous thrombosis occurred before arterial thrombosis and had different risk factors. Events preceding venous thrombosis included cutaneous vasculitis, nephrotic syndrome, and glucocorticoids dosage. In addition, patients with a combination of LAC and anti-RNP/Sm positivity were at risk of venous thrombosis. The ability of this combination to predict venous thrombotic events was greater than that of LAC alone, with a specificity of $96 \%$ and negative predictive value of nearly $89.8 \%$. In contrast, risk factors for arterial thrombotic events were more traditional, including smoking, age, and diabetes [66]. These findings are partially consistent with an earlier cohort study of 544 patients in whom the overall rate of thrombotic events was also $16 \%$, but a higher proportion of events were arterial (11 vs. $5 \%$ ). Most of the events occurred in the first 5 years, with an increased risk in the first year. Patients with thrombotic events had higher baseline and average disease activity, used more antihypertensive drugs, and had higher smoking rates [67].

\section{Thrombotic Microangiopathy and Rheumatological Conditions}

Thrombotic microangiopathy (TMA) comprises several clinical conditions, all characterized by microangiopathic hemolytic anemia (MAHA), thrombocytopenia, and end-organ ischemia. It involves multiple organs, such as the kidneys, lungs, and central nervous system, and often presents as a medical emergency [68]. TMA may be primary or secondary. Primary TMAs, such as thrombotic thrombocytopenia purpura and atypical hemolytic uremic syndrome, may go unnoticed until a stressogenic condition such as pregnancy or infection precipitates an acute episode. Secondary TMAs are complications of underlying disorders. Scleroderma renal crisis (SRC), CAPS, and 
SLE are the main rheumatological disorders that can result in life-threatening complications [68].

SRC occurs in $5-10 \%$ of the patients with systemic sclerosis, usually during the course of the disease and is considered a medical emergency. MAHA is present in $43 \%$ of the patients with SRC and is identified by schistocytes in blood smear and mild thrombocytopenia (platelet count above $50,000 / \mathrm{mm}^{3}$ ), which normalizes as blood pressure returns to normal [69]. MAHA can be misdiagnosed as thrombocytopenia purpura and may be differentiated by a normal ADAMST13 level. The risk of SRC is higher in patients with early diffuse cutaneous disease, recent use of glucocorticoids (daily prednisone dose $\geq 15$ $\mathrm{mg}$ ), and anti-RNA polymerase 3 antibodies [69, 70]. Other predictive factors are recent-onset anemia and cardiac events such as pericardial effusion and congestive heart failure [71]. SRC is manifested clinically by elevated blood pressure, oliguric renal failure with microscopic hematuria, non-nephrotic-range proteinuria, and thrombocytopenia; symptoms include headache, blurred vision, seizures, and encephalopathy. Pulmonary edema, pericarditis, and myocarditis may also be present and suggest a poor prognosis [71].

The definition and classification of SRC are complex owing to the wide range in severity of the clinical picture, from slight elevation in arterial pressure and mild deterioration in renal function to hypertension crisis and rapidly progressive renal failure. Indeed, $10 \%$ of the affected patients are normotensive [72], although their blood pressure is still elevated compared to their usual values [71]. In an attempt to establish a core set of items for the classification of SRC, an international, multidisciplinary panel of experts recommended the following consensus criteria: elevation of blood pressure, kidney injury, and renal histopathology consistent with SRC, MAHA, and target-organ dysfunction including hypertensive retinopathy, hypertensive encephalopathy, acute heart failure, and acute pericarditis [72]. In cases with an atypical clinical presentation, renal biopsy can assist in making the diagnosis, as it can show characteristic changes in arteries and arterioles $[69,71]$.

Immediately upon diagnosis of SRC, angiotensin-converting enzyme (ACE) inhibitors such as captopril should be started in high dose because this is the only treatment shown to improve outcome and even to benefit survival. However, the evidence is based on prospective studies, as randomized clinical trials are lacking and most likely will not be performed. The treatment should be continued for as long as there is still a chance for renal function to improve. There are no data to support prophylactic treatment with ACE inhibitors [73]. Although the prognosis of SRC

Hematological Manifestations in Rheumatic Diseases has improved with the introduction of ACE inhibitors, mortality rates remain high at more than $30 \%$ at 1 year. Of the survivors, $25 \%$ will require permanent dialysis [72].

Primary APS is defined by thrombotic events, either arterial or venous, or obstetrical complications, in the presence of antiphospholipid antibodies without evidence of an underlying condition. In addition to these known criteria, patients with APS may have manifestations of microvascular injury in the kidneys, nervous system, heart valves, skin, or other organs [70]. CAPS is the most devastating complication of APS, occurring in less than $1 \%$ of the patients. It presents with multi-organ thrombosis. Although both large and small blood vessels may be involved, in about $16 \%$ of the cases, small-vessel thrombosis is the main clinical event, making the presentation identical to other thrombotic microangiopathies. Indeed, some studies suggested that CAPS should be included among the thrombotic microangiopathies [33]. In these cases, the accurate diagnosis of CAPS is challenging since up to $12 \%$ of the healthy adults are positive for antiphospholipid antibodies, and their pathogenic role is questionable. Sometimes, a precipitating condition precedes CAPS, such as infection, surgery, medications, or anticoagulation withdrawal. Patients presenting with TMA and positive antiphospholipid antibodies should undergo thorough workup to exclude underlying conditions such as infection, malignancy, and pregnancy, as well as other TMAs such as thrombocytopenic purpura, hemolytic uremic syndrome, and disseminated intervascular coagulation [74]. A set of classification criteria were proposed at the 10th International Congress on Antiphospholipid Antibodies as follows: involvement of 3 or more organs or systems; development of manifestations during less than 1 week; laboratory confirmation of the presence of LAC and/or anti-cardiolipin antibodies; and histopathologic evidence of small-vessel occlusion in at least 1 organ. The 14th International Congress proposed that the histopathology should be substituted with exclusion of other diagnoses since biopsy is not always available in these critical patients $[74,75]$. Treatment includes mainly anticoagulation, glucocorticoids, and plasma exchange. IVIG and cyclophosphamide are sometimes used, and case reports suggest treatment with eculizumab. No consensus on standardized treatment exists [76, 77].

Rarely, TMA can be secondary to SLE. In these cases, TMA may go unrecognized because anemia, thrombocytopenia, deteriorating renal function, and fever or confusion may be attributed to SLE flare. In a retrospective comparison of SLE nephritis patients with and without biopsy proven TMA [78], more patients with SLE and re- 
nal TMA required acute hemodialysis at presentation, and they had a more active disease, with lower GFR rates compared to patients with SLE nephritis without TMA. They also had lower C3 levels and more anti-RO seropositivity. There was no difference in age or sex between the 2 groups. TMA in the context of SLE is multifactorial; associations have been reported for autoantibodies to ADAMTS13 and complement activation, as well as medication intake and precedent infections. Therefore, treatment is complex, including plasma exchange, immunosuppression, and sometimes in refractory cases, eculizumab $[71,79,80]$.

\section{Conclusion}

RAD can present with cytopenias, MAHA, or thrombosis at the time of onset or as a LOC. Hematologists should be alert to these associations in order to reach an accurate diagnosis. Treatment should be administered promptly, with rheumatological consultation.

\section{Acknowledgement}

The authors wish to thank Gloria Ginzach for the editorial assistance.

\section{Conflict of Interest Statement}

The authors have no conflicts of interest to declare.

\section{Funding Sources}

The authors did not receive any funding.

\section{Author Contributions}

A. Klein drafted the manuscript; Y. Molad critically revised the manuscript. Both authors approved all versions including the final version and are responsible for the accuracy and integrity of all aspects of the manuscript.

\section{References}

1 Keeling DM, Isenberg DA. Haematological manifestations of systemic lupus erythematosus. Blood Rev. 1993;7(4):199-207.

2 Nossent JC, Swaak AJ. Prevalence and significance of haematological abnormalities in patients with systemic lupus erythematosus. Q J Med. 1991;80(291):605-12.

3 Tan EM, Cohen AS, Fries JF, Masi AT, McShane DJ, Rothfield NF, et al. The 1982 revised criteria for the classification of systemic lupus erythematosus. Arthritis Rheum. 1982 Nov;25(11):1271-7.

4 Petri M, Orbai AM, Alarcón GS, Gordon C, Merrill JT, Fortin PR, et al. Derivation and validation of the Systemic Lupus International Collaborating Clinics classification criteria for systemic lupus erythematosus. Arthritis Rheum. 2012 Aug;64(8):2677-86.

5 Levine AB, Erkan D. Clinical assessment and management of cytopenias in lupus patients. Curr Rheumatol Rep. 2011 Aug; 13(4):291-9.

6 Dias AM, do Couto MC, Duarte CC, Inês LP, Malcata $A B$. White blood cell count abnormalities and infections in one-year follow-up of 124 patients with SLE. Ann N Y Acad Sci. 2009 Sep;1173:103-7.

7 Rivero SJ, Díaz-Jouanen E, Alarcón-Segovia D. Lymphopenia in systemic lupus erythematosus. Clinical, diagnostic, and prognostic significance. Arthritis Rheum. 1978 Apr;21(3): 295-305.

8 Schulze-Koops H. Lymphopenia and autoimmune diseases. Arthritis Res Ther. 2004;6(4): 178-80.

9 Fayyaz A, Igoe A, Kurien BT, Danda D, James JA, Stafford HA, et al. Haematological mani- festations of lupus. Lupus Sci Med. 2015 Mar 3;2(1):e000078.

10 Klein A, Polliack A, Gafter-Gvili A. Systemic lupus erythematosus and lymphoma: incidence, pathogenesis and biology. Leuk Res. 2018 Dec;75:45-9.

11 Beyan E, Beyan C, Turan M. Hematological presentation in systemic lupus erythematosus and its relationship with disease activity. Hematology. 2007;12(3):257-61.

12 Miranda-Hernández D, Cruz-Reyes C, Monsebaiz-Mora C, Gómez-Bañuelos E, Ángeles $\mathrm{U}$, Jara LJ, et al. Active haematological manifestations of systemic lupus erythematosus lupus are associated with a high rate of in-hospital mortality. Lupus. 2017;26(6):640-5.

13 Meyer A, Guffroy A, Blaison G, Dieudonne Y, Amoura Z, Bonnotte B, et al. Systemic lupus erythematosus and neutropaenia: a hallmark of haematological manifestations. Lupus Sci Med. 2020;7(1):e000399.

14 Atallah-Yunes SA, Ready A, Newburger PE. Benign ethnic neutropenia. Blood Rev. 2019; 37:100586

15 Carli L, Tani C, Vagnani S, Signorini V, Mosca M. Leukopenia, lymphopenia, and neutropenia in systemic lupus erythematosus: Prevalence and clinical impact: a systematic literature review. Semin Arthritis Rheum. 2015 Oct; 45(2):190-4.

16 Newman KA, Akhtari M. Management of autoimmune neutropenia in Felty's syndrome and systemic lupus erythematosus. Autoimmun Rev. 2011 May;10(7):432-7.

17 Hellmich B, Schnabel A, Gross WL. Treatment of severe neutropenia due to Felty's syn- drome or systemic lupus erythematosus with granulocyte colony-stimulating factor. Semin Arthritis Rheum. 1999 Oct;29(2):82-99.

18 Owlia MB, Newman K, Akhtari M. Felty's syndrome, insights and updates. Open Rheumatol J. 2014;8:129-36.

19 Jain T, Mittal C, Sengupta R, Rubin B. Nonarticular Felty's syndrome: an uncommon diagnosis. Neth J Med. 2015 Nov;73(9):435-6.

20 Bowman SJ. Hematological manifestations of rheumatoid arthritis. Scand J Rheumatol. 2002;31(5):251-9.

21 Balint GP, Balint PV. Felty's syndrome. Best Pract Res Clin Rheumatol. 2004 Oct;18(5): 631-45.

22 Narváez J, Domingo-Domenech E, GómezVaquero C, López-Vives L, Estrada P, Aparicio $\mathrm{M}$, et al. Biological agents in the management of Felty's syndrome: a systematic review. Semin Arthritis Rheum. 2012 Apr; 41(5):658-68.

23 Heylen L, Dierickx D, Vandenberghe P, Westhovens R. Targeted herapy with rituximab in Felty's syndrome: a case report. Open Rheumatol J. 2012;6:312-4.

24 Lee EJ, Lee AI. Thrombocytopenia. Prim Care. 2016 Dec;43(4):543-57.

25 Fernández M, Alarcón GS, Apte M, Andrade RM, Vilá LM, Reveille JD. Systemic lupus erythematosus in a multiethnic US cohort: XLIII. The significance of thrombocytopenia as a prognostic factor. Arthritis Rheum. 2007 Feb; 56(2):614-21.

26 Kado R, McCune WJ. Treatment of primary and secondary immune thrombocytopenia. Curr Opin Rheumatol. 2019;31(3):213-22. 
27 Arnal C, Piette JC, Léone J, Taillan B, Hachulla E, Roudot-Thoraval F, et al. Treatment of severe immune thrombocytopenia associated with systemic lupus erythematosus: 59 cases. J Rheumatol. 2002 Jan;29(1):75-83.

28 Newman K, Owlia MB, El-Hemaidi I, Akhtari $\mathrm{M}$. Management of immune cytopenias in patients with systemic lupus erythematosus: old and new. Autoimmun Rev. 2013;12(7):78491.

29 Cobo-Ibáñez T, Loza-Santamaría E, PegoReigosa JM, Marqués AO, Rúa-Figueroa I, Fernández-Nebro A, et al. Efficacy and safety of rituximab in the treatment of non-renal systemic lupus erythematosus: a systematic review. Semin Arthritis Rheum. 2014 Oct; 44(2):175-85.

30 Serris A, Amoura Z, Canouï-Poitrine F, Terrier B, Hachulla E, Costedoat-Chalumeau N, et al. Efficacy and safety of rituximab for systemic lupus erythematosus-associated immune cytopenias: a multicenter retrospective cohort study of 71 adults. Am J Hematol. 2018 Mar;93(3):424-9.

31 Shobha V, Sanil S, Roongta R. Eltrombopag: efficacy and safety in steroid refractory lupusassociated immune thrombocytopenia. J Clin Rheumatol. 2020 Oct;26(7):274-8.

32 Lusa A, Carlson A. Safety and efficacy of thrombopoeitin mimetics for refractory immune thrombocytopenia purpura in patients with systemic lupus erythematosus or antiphospholipid syndrome: a case series. Lupus. 2018 Sep;27(10):1723-8.

33 Uthman I, Godeau B, Taher A, Khamashta M. The hematologic manifestations of the antiphospholipid syndrome. Blood Rev. 2008 Jul;22(4):187-94.

34 Rattarittamrong E, Eiamprapai P, Tantiworawit A, Rattanathammethee T, Hantrakool S, Chai-Adisaksopha C, et al. Clinical characteristics and long-term outcomes of warm-type autoimmune hemolytic anemia. Hematology. 2016 Jul;21(6):368-74.

35 Costallat GL, Appenzeller S, Costallat LT. Evans syndrome and systemic lupus erythematosus: clinical presentation and outcome. Joint Bone Spine. 2012 Jul;79(4):362-4.

36 Velo-García A, Castro SG, Isenberg DA. The diagnosis and management of the haematologic manifestations of lupus. J Autoimmun. 2016 Nov;74:139-60.

37 Gormezano NW, Kern D, Pereira OL, Esteves GC, Sallum AM, Aikawa NE, et al. Autoimmune hemolytic anemia in systemic lupus erythematosus at diagnosis: differences between pediatric and adult patients. Lupus. 2017 Apr;26(4):426-30.

38 Skare T, Picelli L, Dos Santos TAG, Nisihara R. Direct antiglobulin (Coombs) test in systemic lupus erythematosus patients. Clin Rheumatol. 2017 Sep;36(9):2141-4.

39 Go RS, Winters JL, Kay NE. How I treat autoimmune hemolytic anemia. Blood. 2017 Jun 1;129(22):2971-9.
40 Reynaud Q, Durieu I, Dutertre M, Ledochowski S, Durupt S, Michallet AS, et al. Efficacy and safety of rituximab in auto-immune hemolytic anemia: a meta-analysis of 21 studies. Autoimmun Rev. 2015 Apr;14(4): 304-13.

41 Rajakulendran S, Gadsby K, Allen D, O’Reilly S, Deighton C. Neutropenia while receiving anti-tumour necrosis factor treatment for rheumatoid arthritis. Ann Rheum Dis. 2006 Dec;65(12):1678-9.

42 Hastings R, Ding T, Butt S, Gadsby K, Zhang W, Moots RJ, et al. Neutropenia in patients receiving anti-tumor necrosis factor therapy. Arthritis Care Res. 2010 Jun;62(6):764-9.

43 Azevedo VF, Silva MB, Marinello DK, Santos FD, Silva GB. Leukopenia and thrombocytopenia induced by etanercept: two case reports and literature review. Rev Bras Reumatol. 2012 Jan-Feb;52(1):110-2.

$44 \mathrm{He} \mathrm{Y,} \mathrm{Sawalha} \mathrm{AH.} \mathrm{Drug-induced} \mathrm{lupus} \mathrm{ery-}$ thematosus: an update on drugs and mechanisms. Curr Opin Rheumatol. 2018;30(5): 490-7.

45 Ramos-Casals M, Roberto-Perez-Alvarez R, Diaz-Lagares C, Cuadrado MJ, Khamashta MA; BIOGEAS Study Group. Autoimmune diseases induced by biological agents: a double-edged sword? Autoimmun Rev. 2010 Jan; 9(3):188-93.

46 Salmon JH, Cacoub P, Combe B, Sibilia J, Pallot-Prades B, Fain O, et al. Late-onset neutropenia after treatment with rituximab for rheumatoid arthritis and other autoimmune diseases: data from the AutoImmunity and Rituximab Registry. RMD Open. 2015 Jun 30; 1(1):e000034.

47 Monaco WE, Jones JD, Rigby WF. Rituximab associated late-onset neutropenia: a rheumatology case series and review of the literature. Clin Rheumatol. 2016 Oct;35(10):2457-62.

48 Tesfa D, Ajeganova S, Hägglund H, Sander B, Fadeel B, Hafström I, et al. Late-onset neutropenia following rituximab therapy in rheumatic diseases: association with B lymphocyte depletion and infections. Arthritis Rheum. 2011 Aug;63(8):2209-14.

49 Schulze-Koops H, Strand V, Nduaka C, DeMasi R, Wallenstein G, Kwok K, et al. Analysis of haematological changes in tofacitinibtreated patients with rheumatoid arthritis across phase 3 and long-term extension studies. Rheumatology. 2017 Jan;56(1):46-57.

50 Ogata A, Kato Y, Higa S, Yoshizaki K. IL-6 inhibitor for the treatment of rheumatoid arthritis: a comprehensive review. Mod Rheumatol. 2019 Mar;29(2):258-67.

51 Emmi G, Silvestri E, Squatrito D, Amedei A, Niccolai E, D'Elios MM, et al. Thrombosis in vasculitis: from pathogenesis to treatment. Thromb J. 2015 Apr 16;13:15.

52 Katz OB, Brenner B, Horowitz NA. Thrombosis in vasculitic disorders-clinical manifestations, pathogenesis and management. Thromb Res. 2015 Sep;136(3):504-12.
53 Morgan MD, Turnbull J, Selamet U, KaurHayer M, Nightingale P, Ferro CJ, et al. Increased incidence of cardiovascular events in patients with antineutrophil cytoplasmic antibody-associated vasculitides: a matchedpair cohort study. Arthritis Rheum. 2009 Nov;60(11):3493-500.

54 Allenbach Y, Seror R, Pagnoux C, Teixeira L, Guilpain P, Guillevin L, et al. High frequency of venous thromboembolic events in ChurgStrauss syndrome, Wegener's granulomatosis and microscopic polyangiitis but not polyarteritis nodosa: a systematic retrospective study on 1130 patients. Ann Rheum Dis. 2009 Apr;68(4):564-7.

55 Stassen PM, Derks RP, Kallenberg CG, Stegeman CA. Venous thromboembolism in ANCA-associated vasculitis: incidence and risk factors. Rheumatology. 2008 Apr;47(4):5304.

56 Gonzalez-Gay MA, Vazquez-Rodriguez TR, Gomez-Acebo I, Pego-Reigosa R, Lopez-Diaz MJ, Vazquez-Triñanes MC, et al. Strokes at time of disease diagnosis in a series of $287 \mathrm{pa}-$ tients with biopsy-proven giant cell arteritis. Medicine. 2009 Jul;88(4):227-35.

57 Duarte MM, Geraldes R, Sousa R, Alarcão J, Costa J. Stroke and transient ischemic attack in Takayasu's arteritis: a systematic review and meta-analysis. J Stroke Cerebrovasc Dis. 2016 Apr;25(4):781-91.

58 Aviña-Zubieta JA, Bhole VM, Amiri N, Sayre EC, Choi HK. The risk of deep venous thrombosis and pulmonary embolism in giant cell arteritis: a general population-based study. Ann Rheum Dis. 2016 Jan;75(1):148-54.

59 Unizony S, Lu N, Tomasson G, Zhang Y, Merkel PA, Stone JH, et al. Temporal trends of venous thromboembolism risk before and after diagnosis of giant cell arteritis. Arthritis Rheumatol. 2017 Jan;69(1):176-84.

60 Chung WS, Peng CL, Lin CL, Chang YJ, Chen YF, Chiang JY, et al. Rheumatoid arthritis increases the risk of deep vein thrombosis and pulmonary thromboembolism: a nationwide cohort study. Ann Rheum Dis. 2014 Oct; 73(10): 1774-80.

61 Matta F, Singala R, Yaekoub AY, Najjar R, Stein PD. Risk of venous thromboembolism with rheumatoid arthritis. Thromb Haemost. 2009 Jan; 101(1):134-8

62 Holmqvist ME, Neovius M, Eriksson J, Mantel Ä, Wållberg-Jonsson S, Jacobsson LT, et al. Risk of venous thromboembolism in patients with rheumatoid arthritis and association with disease duration and hospitalization. JAMA. 2012 Oct 3;308(13):1350-6.

63 Ogdie A, Kay McGill N, Shin DB, Takeshita J, Jon Love $\mathrm{T}$, Noe $\mathrm{MH}$, et al. Risk of venous thromboembolism in patients with psoriatic arthritis, psoriasis and rheumatoid arthritis: a general population-based cohort study. Eur Heart J. 2018 Oct 14;39(39):3608-14.

64 Erkan D. Lupus and thrombosis. J Rheumatol. 2006 Sep;33(9):1715-7.
Hematological Manifestations in Rheumatic Diseases
Acta Haematol 2021;144:403-412

DOI: $10.1159 / 000511759$ 
65 Bazzan M, Vaccarino A, Marletto F. Systemic lupus erythematosus and thrombosis. Thromb J. 2015 Apr 23;13:16.

66 Hinojosa-Azaola A, Romero-Diaz J, VargasRuiz AG, Nuñez-Alvarez CA, Cicero-Casarrubias A, Ocampo-Torres MC, et al. Venous and arterial thrombotic events in systemic lupus erythematosus. J Rheumatol. 2016 Mar; 43(3):576-86.

67 Sarabi ZS, Chang E, Bobba R, Ibanez D, Gladman $\mathrm{D}$, Urowitz $\mathrm{M}$, et al. Incidence rates of arterial and venous thrombosis after diagnosis of systemic lupus erythematosus. Arthritis Rheum. 2005 Aug 15;53(4):609-12.

68 George JN, Nester CM. Syndromes of thrombotic microangiopathy. N Engl J Med. 2014 Aug 14;371(19): 1847-8.

69 Mouthon L, Bussone G, Berezné A, Noël LH, Guillevin L. Scleroderma renal crisis. J Rheumatol. 2014 Jun;41(6):1040-8.

70 Babar F, Cohen SD. Thrombotic microangiopathies with rheumatologic involvement. Rheum Dis Clin North Am. 2018 Nov;44(4): $635-49$.
71 Bose N, Chiesa-Vottero A, Chatterjee S. Scleroderma renal crisis. Semin Arthritis Rheum. 2015 Jun;44(6):687-94.

72 Butler EA, Baron M, Fogo AB, Frech T, Ghossein C, Hachulla E, et al. Scleroderma Clinical Trials Consortium Scleroderma Renal Crisis Working Group. Generation of a core set of items to develop classification criteria for scleroderma renal crisis using consensus methodology. Arthritis Rheumatol. 2019 Jun; 71(6):964-71.

73 Kowal-Bielecka O, Fransen J, Avouac J, Becker M, Kulak A, Allanore Y, et al. Update of EULAR recommendations for the treatment of systemic sclerosis. Ann Rheum Dis. 2017 Aug;76(8):1327-39.

74 Rodríguez-Pintó I, Espinosa G, Cervera R. Catastrophic APS in the context of other thrombotic microangiopathies. Curr Rheumatol Rep. 2015 Jan;17(1):482.

75 Asherson RA, Cervera R, de Groot PG, Erkan D, Boffa MC, Piette JC, et al. Catastrophic Antiphospholipid Syndrome Registry Project Group. Catastrophic antiphospholipid syndrome: international consensus statement on classification criteria and treatment guidelines. Lupus. 2003;12(7):530-4.
76 Ortel TL, Erkan D, Kitchens CS. How I treat catastrophic thrombotic syndromes. Blood. 2015 Sep 10;126(11):1285-93.

77 Strakhan M, Hurtado-Sbordoni M, Galeas N, Bakirhan K, Alexis K, Elrafei T. 36-year-old female with catastrophic antiphospholipid syndrome treated with eculizumab: a case report and review of literature. Case Rep Hematol. 2014;2014:704371.

78 Li C, Yap C, Chan G, Wen YB, Li H, Tang C, et al. Clinical outcomes and clinico-pathological correlations in lupus nephritis with kidney biopsy showing thrombotic microangiopathy. J Rheumatol. 2019 Nov;46(11):147884.

79 Chen $\mathrm{MH}$, Chen $\mathrm{MH}$, Chen WS, Mu-Hsin Chang P, Lee HT, Lin HY, et al. Thrombotic microangiopathy in systemic lupus erythematosus: a cohort study in North Taiwan. Rheumatology. 2011 Apr;50(4):768-75.

80 El-Husseini A, Hannan S, Awad A, Jennings S, Cornea V, Sawaya BP. Thrombotic microangiopathy in systemic lupus erythematosus: efficacy of eculizumab. Am J Kidney Dis. 2015 Jan;65(1):127-30. 